\title{
Shape change analysis of tensegrity models
}

\author{
Chai Lian $\mathrm{Oh}^{\mathrm{a}}$ \\ Kok Keong Choong ${ }^{b^{*}}$ (D) \\ Toku Nishimurac ${ }^{c}$ \\ Jae-Yeol Kim ${ }^{d}$

\section{Omid Hassanshahi ${ }^{b}$}

a Faculty of Civil Engineering, Universiti Teknologi MARA, 40450 Shah Alam, Selangor, Malaysia. E-mail: chailian_080@yahoo.com ${ }^{b}$ School of Civil Engineering, Engineering Campus, Universiti Sains Malaysia, 14300 Nibong Tebal, Penang, Malaysia. E-mail: cekkc@usm.my, omid.hasanshahi@gmail.com

c Department of Architectural Engineering, Institute of Disaster and Environmental Science, Kanazawa Institute of Technology, Ishikawa, Japan. E-mail: eigen-t.nishimura@neptune.kanazawa-it.ac.jp

d Department of Architectural Engineering, Hyupsung University, Hwaseong-si, Gyeongi-do, Republic of Korea. E-mail:

jjaerinim@empas.com

${ }^{*}$ Corresponding author

https://doi.org/10.1590/1679-78255407

\begin{abstract}
This paper presents a computational strategy for shape change of tensegrity models to achieve the prescribed target coordinates of a set of monitored nodes via forced elongation of cables. Mathematical formulation for incremental equilibrium equations of a tensegrity model during the shape change analysis is derived and presented. An optimization approach in determining forced elongation of cables using sequential quadratic programming with defined inequality constraints is formulated and presented. Four tensegrity models namely the simplex, quadruplex, two-stage tensegrity model and tapered three-stage tensegrity model are tested using the proposed shape change algorithm. Capability of tensegrity models to undergo bending, axial, twisting deformation and combinations of these deformations is also described.
\end{abstract}

\section{Keywords}

Tensegrity, shape change, SQP, forced elongation.

\section{INTRODUCTION}

There has been a growing interest in the shape change of tensegrities since 1990s. Tensegrities can be folded or unfolded through changes in their member lengths. Features such as simple joints (i.e. pinned jointed) and controllable cables (through actuators and sensors installed in cables) in a tensegrity are advantageous for the structure to undergo shape change. Stability of tensegrity is maintained through the introduction of self-equilibrated force mode. Due to the nature of interaction of only compression and tension forces through strut and cable members to maintain stability, there is potential for tensegrity to convert any local pressure into global deformation and subsequently seek another balanced configuration in tensegrity. This feature makes tensegrity a suitable system to be considered for shape change studies. 
Shape change of tensegrities have been studied and applied especially for the design of deployable structures and robots. Specifically, the use of tensegrities as deployable structures has been proposed for space applications (Fazli and Abedian, 2011, Dalilsafaei et al., 2012), deployable beams (Averseng et al., 2012), deployable support for solar energy harvesting on water canals (Carpentieri et al., 2017), deployable footbridge (Rhode-Barbarigos et al., 2012, Veuve et al., 2016), composite solar façades and wind generators (Cimmino et al., 2017), and compressive structures (Skelton and Oliveira, 2010). Furuya (1992) was probably the first researcher to use tensegrity as a deployable structure even though the investigation was at the conceptual level. A proper deployment procedure of tensegrities begun with the concept of cable control by Sultan and Skelton $(1998,2003)$. An optimization method to determine the reference trajectory for the deployment of a tensegrity model was developed by van de Wijdeven and de Jager (2005). The deployment control of tensegrity with minimal energy was investigated numerically by Li, et al. (2011). On the other hand, tensegrity-based robots have been suggested as co-robots (Kim et al., 2014), locomotors (Du et al., 2016) and manipulators (Moored et al., 2011). Recent studies on tensegrity robots mainly focused on the design, control strategies and gaits production (i.e. forward, crawling and rolling) of the robots (Kim et al., 2014, Moored et al., 2011). An idea of using tensegrities as inspection robotic tools to handle difficult environments in construction industry has been a motivation to the study (Figure 1) (Oh et al., 2012). However, the numerical formulation for simulating the shape change for the tensegrities was not shown.

Generally, shape change strategies of the tensegrities have been classified as passive and active deployment schemes (Tur and Juan, 2009). The former utilizes the external forces whereas the later actuates the length of the elements to allow for shape change in tensegrities. Most of the studies employed active deployment strategy in achieving shape change of tensegrities. For instance, Zhang et al. proposed a cable-controlled reconfiguration procedure to change an initial configuration of prismatic tensegrity to a new symmetrical configuration (Zhang et al., 2014). Equal length adjustments in a shortening manner were applied to the defined additional cables for the reconfiguration of tensegrities. Caluwaerts and Carbajal presented optimization methods for reconfiguration of the tensegrities via tuning of stored energy and stiffness (Caluwaerts and Carbajal, 2015). An example of a 6-bar tensegrity to move from an initial shape to the target shape was achieved through active cables with the alteration of stored elastic energy. Veuve et al. investigated a control methodology to connect two half bridges through the changes of cable length (Veuve et al., 2016). The deployment of the footbridges was solved based on the distance between a pair of nodal coordinates of two mid-span connectors. Most of these deployment strategies modified the stored energy and stiffness for the shape change of tensegrities. In this study, the capability of tensegrity to achieve a target position defined by the target coordinates of selected joints through advancement of the shape from the original until the final position is examined (Figure 2).

This paper presents an incremental analysis procedure for shape change of tensegrities. In this paper, the shape change of a tensegrity is used to describe a process of achieving a prescribed target position defined by coordinates of a given set of target joints of the tensegrity. The aims of this paper are twofold: (i) to propose a method for shape change analysis of tensegrities and (ii) to demonstrate the shape change capability of tensegrity through the investigation of the deformed configurations. The shape change process of tensegrity is realized through forced elongation of cables. An optimization procedure to determine the forced elongation of cables in each incremental step is presented. A total of four tensegrity models (i.e. simplex, quadruplex, two-stage and tapered three stage tensegrity models) were used to verify the applicability of the proposed shape change algorithm.

This paper consists of five sections. Section 2 defines the terminology used in the shape change analysis, presents the incremental equilibrium equations and the proposed optimization method used for shape change analysis. Section 3 describes the proposed method for shape change analysis. Section 4 discusses and evaluates the results of shape change analysis using the proposed method in terms of convergence results and shape change configurations. Finally, Section 5 presents the conclusions. 


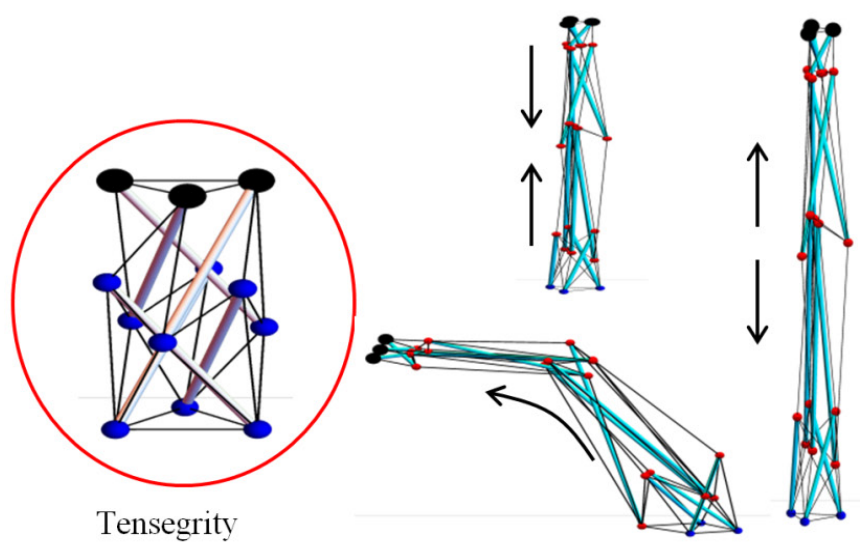

Shape change possibilities

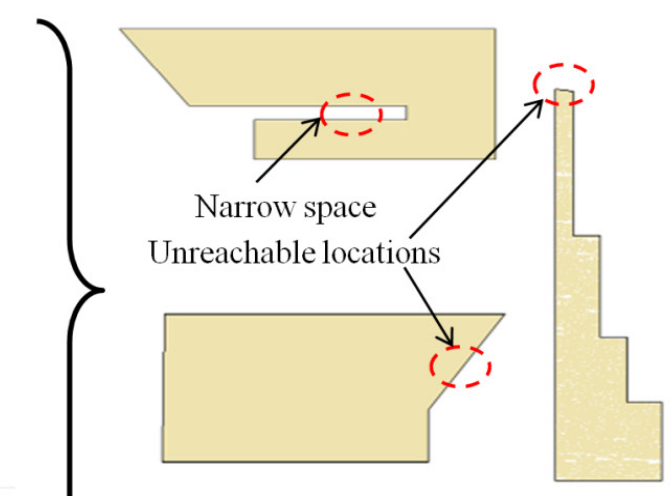

Applications - aiding to reach difficult situations in construction industry

Figure 1: Shape change applications of tensegrity.

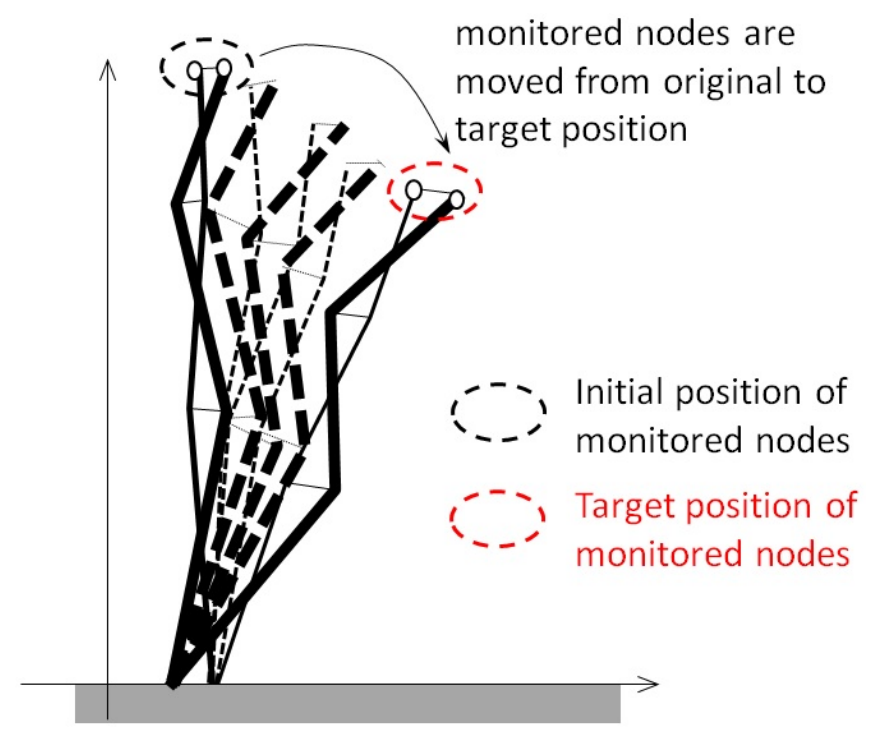

Figure 2: A tensegrity undergoing shape change from original to achieve final position.

\section{SHAPE CHANGE OF TENSEGRITY MODELS}

\subsection{Terminology}

The complete four states involved in shape change analysis in this study are - the initial state, deformed state, shape change state and target state. Figure 3 illustrates these four states involving the example of a self-equilibrated two-stage tensegrity tower. At initial state, the tower is in self-equilibrium and stands freely without constraints and external forces. At deformed state, support condition in the form of pinned support is specified and the tower experiences linear elastic deformation under self-weight loading condition. At shape change state, the tower undergoes shape change by means of forced elongation of cables. Finally, at target state which is the final state at the end of the shape change analysis, the monitored nodes reach the prescribed target coordinates. The computational strategy proposed in this paper is specifically for the shape change state to achieve the target state. 


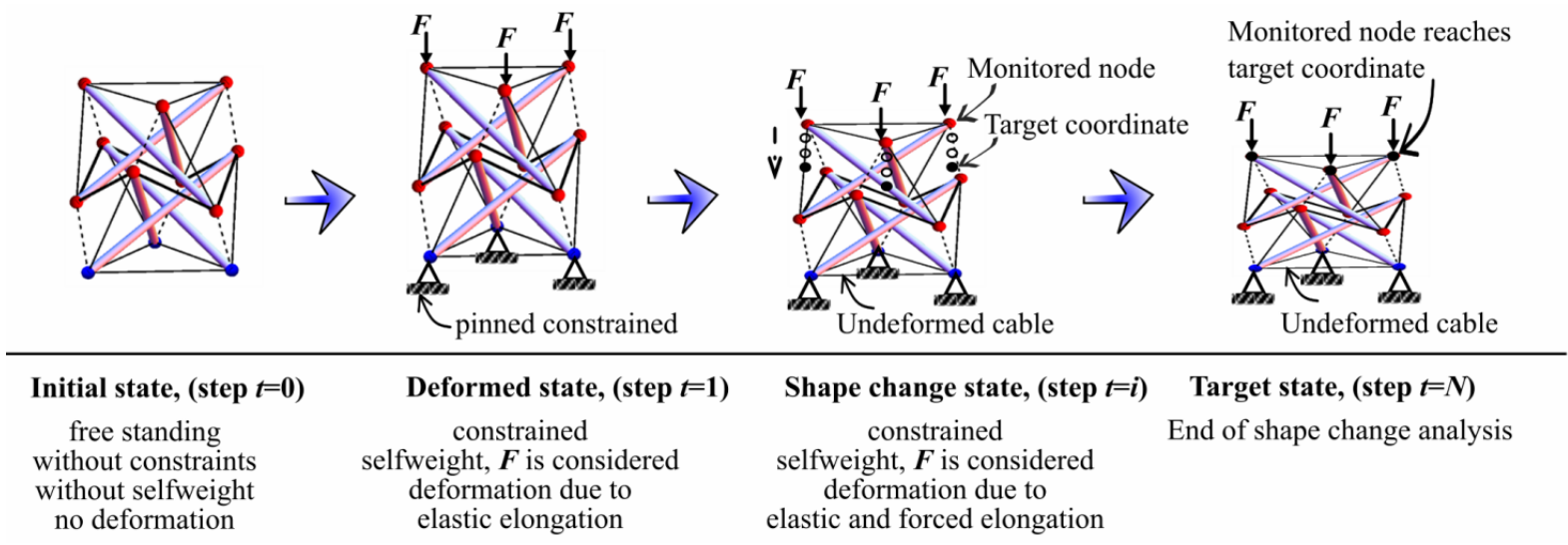

Figure 3: Four states in shape change analysis.

Consider an element $k(k=1,2, \ldots, m ; m=$ total number of elements) of a tensegrity model which connects node $i$ and $j(i<j)$ in a Cartesian coordinate system $O-X Y Z$ as shown in Figure 4. The equilibrium configurations of element $k$ are evaluated at discrete step $t=0$ (initial state), step $t=1$ (deformed state), step $t=i$ (shape change state, with $i=2$, $\ldots, N$ ), step $t=N$ (target state) where $N$ is the total number of incremental step in shape change analysis. Nodal coordinates at node $i$ and $j$ associated with element $k$ at step 0 , step 1, step $i$ and step $N$ are denoted as ${ }^{0} \boldsymbol{x}_{i}^{\top},{ }^{\top} \boldsymbol{x}_{i}{ }^{\top}, \boldsymbol{x}_{i}{ }^{\top},{ }^{N} \boldsymbol{x}_{i}{ }^{\top}$ and ${ }^{0} \boldsymbol{x}_{j}^{\top},{ }^{1} \boldsymbol{x}_{j}^{\top},{ }^{\prime} \boldsymbol{x}_{j}{ }^{\top},{ }^{N} \boldsymbol{x}_{j}^{\top}$, respectively, where left superscripts refer to the shape change state of element $k$. Also shown in Figure 3 are elastic elongation $u$, length increment due to elastic elongation $\dot{u}$ and forced elongation $i$, of an element $k$ at the particular step as indicated in the left superscripts. Forced elongation is the allowable change in length apart from elastic elongation of element $k$. For undeformed cable which is cable that is constrained at both ends (see Figure 3$)$, the forced elongation is null $(i=0)$ since the length of the cable remains constant throughout the shape change analysis.

As mentioned earlier, for the shape change analysis considered in this study, a number of nodes are chosen from the unconstrained nodes of a tensegrity model to be assigned as monitored nodes. These monitored nodes are required to reach a set of prescribed target coordinates $\overline{\boldsymbol{x}}$ in certain directional modes. Figure 5 explains the terminology of nodal coordinates of monitored nodes during the shape change analysis. Nodal coordinates of the monitored nodes at previous and current step during shape change analysis are denoted as ${ }^{t-1} x^{\prime}$ and ${ }^{t} x^{\prime}$, respectively. Left superscripts $t-1$ and $t$ which are also used in other expressions in this paper represents the previous and current steps in the shape change analysis, respectively.

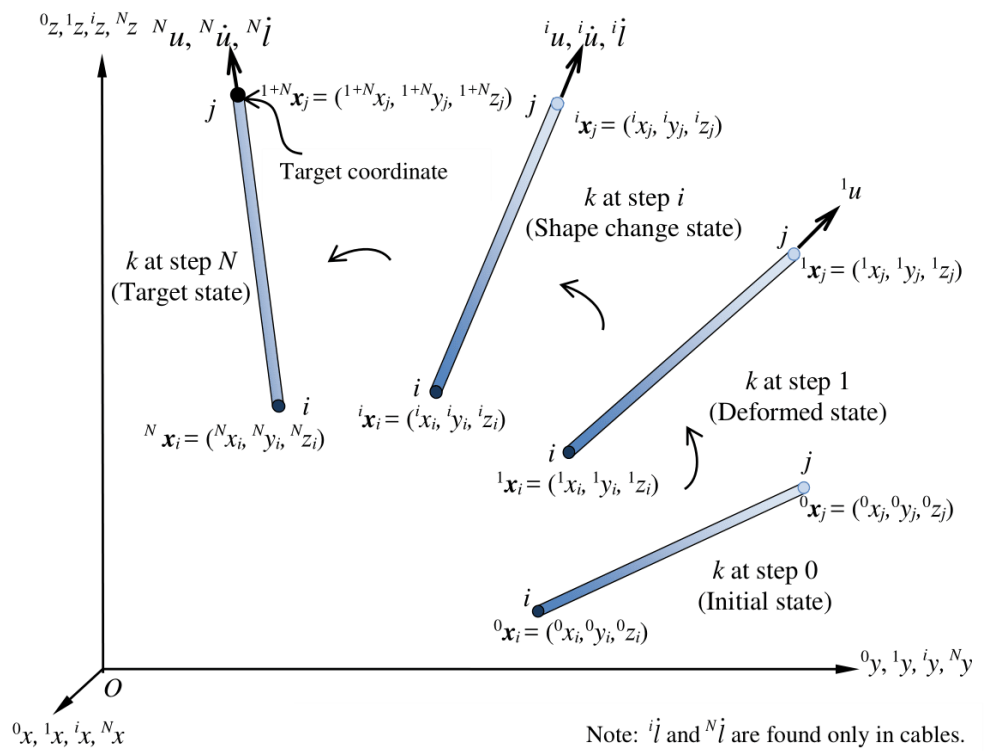

Figure 4: Configurations of element $k$ of tensegrity model during shape change analysis. 


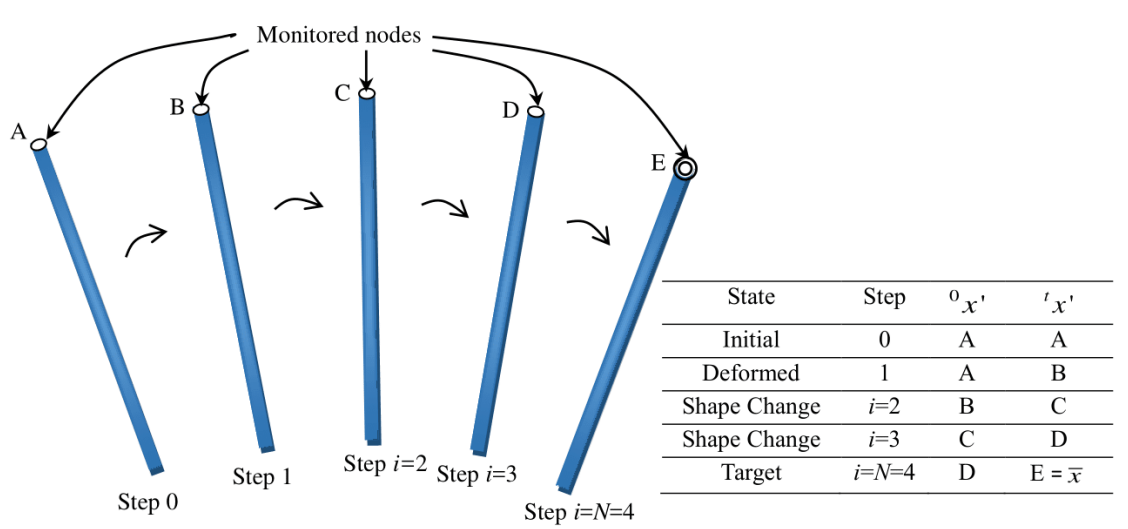

Figure 5: Example on nodal coordinates of monitored nodes at previous and current step.

\subsection{Incremental equilibrium equations}

Equilibrium equation for tensegrity model could be derived based on the basic formulations of pin-jointed structures. For a tensegrity model with $m$ elements, $n$ nodes, $n_{c}$ constraints and $n_{u}\left(3 n-n_{c}\right)$ unconstrained degree of freedoms, the static equilibrium equation for the entire structure could be expressed as follows:

$$
\boldsymbol{F}=\boldsymbol{B n}
$$

where $\boldsymbol{B}$ is a $n_{u} \times m$ matrix consisting of vector of directional cosines $\lambda$ of all elements in the tensegrity model with respect to $x, y, z$ axes, $\boldsymbol{F}$ is a vector of nodal forces with size $n_{u}$ and $\boldsymbol{n}$ is a vector of element axial forces with size $m$. In the incremental analysis for shape change of tensegrity, we assume that the configurations at step $2,3, \ldots,(t-1)$ have already been determined. At the beginning of incremental step $t$, the vector of directional cosine $\boldsymbol{\lambda}$ for a particular member of a tensegrity model could be represented as follows:

$$
{ }^{t} \lambda=\frac{\left({ }^{t-1} \boldsymbol{x}_{j}-{ }^{t-1} \boldsymbol{x}_{i}\right)}{{ }^{t-1} l+{ }^{t-1} u}
$$

where $I, u, \boldsymbol{x}_{i}$ and $\boldsymbol{x}_{j}$ are length after forced elongation, elastic elongation, nodal coordinate at nodes $i$ and $j$, respectively which are taken specifically from the previous step $t-1$ which provides the initial geometrical inputs for the current step of shape change. The compatibility condition for each member at arbitrary time is expressed as

$$
l+u=\sqrt{\left(\boldsymbol{x}_{j}-\boldsymbol{x}_{i}\right)^{T}\left(\boldsymbol{x}_{j}-\boldsymbol{x}_{i}\right)}
$$

Differentiating Equation (2b) with respect to time $t$, one obtains

$$
{ }^{t} \dot{l}+{ }^{t} \dot{u}=\left[\begin{array}{ll}
-{ }^{t} \lambda^{T} & { }^{t} \lambda^{T}
\end{array}\right]\left\{\begin{array}{c}
{ }^{t} \dot{\boldsymbol{x}}_{i} \\
{ }^{t} \dot{\boldsymbol{x}}_{j}
\end{array}\right\}
$$

where ${ }^{t} \boldsymbol{i},{ }^{t} \dot{\boldsymbol{u}},{ }^{t} \dot{\boldsymbol{x}}$ denote incremental forced elongation, incremental elastic elongation and incremental nodal coordinates at step $t$. Nodal coordinates and axial force of element $k$ at step $t$ could be defined as

$$
\begin{aligned}
& { }^{t} \boldsymbol{x}={ }^{t-1} \boldsymbol{x}+{ }^{t} \dot{\boldsymbol{x}} \\
& { }^{t} n={ }^{t-1} n+{ }^{t} \dot{n}
\end{aligned}
$$

where ${ }^{t} \dot{n}$ is the incremental axial force at step $t$. We first derive the incremental nodal coordinate ${ }^{t} \dot{\boldsymbol{x}}$ which corresponds to the elastic elongation for an element $k$ at shape change state. 
Assuming that the element is linear elastic with axial stiffness $E A$ (where $E$ is Young's modulus and $A$ is crosssectional area), known length ${ }^{t-1} l$ and elastic elongation ${ }^{t-1} u$, differentiation of the constitutive relation as shown in Equation (4a):

$$
{ }^{t} n=E A\left(\frac{{ }^{t} u}{{ }^{t-1} l}\right)
$$

will yield the following equation for incremental axial force:

$$
{ }^{t} \dot{n}=E A\left(\frac{{ }^{t} \dot{u}}{{ }^{t-1} l}-\frac{{ }^{t-1} u^{t} \dot{l}}{{ }^{t-1} l^{2}}\right)
$$

The set of incremental equilibrium equation for the entire structure could be obtained by differentiation of the equilibrium equation as shown in Equation (1) as follows:

$$
{ }^{t} \dot{\boldsymbol{F}}={ }^{t} \dot{\boldsymbol{B}}^{t} \boldsymbol{n}+{ }^{t} \boldsymbol{B}^{t} \dot{\boldsymbol{n}}
$$

The incremental equation for each member could be expressed in matrix form as follows:

$$
\left\{\begin{array}{c}
{ }^{t} \dot{\boldsymbol{f}}_{i} \\
{ }^{t} \dot{\boldsymbol{f}}_{j}
\end{array}\right\}=\left[\begin{array}{c}
-{ }^{t} \dot{\boldsymbol{\lambda}} \\
{ }^{t} \dot{\boldsymbol{\lambda}}
\end{array}\right]{ }^{t} n+\left[\begin{array}{c}
-{ }^{t} \boldsymbol{\lambda} \\
{ }^{t} \boldsymbol{\lambda}
\end{array}\right]{ }^{t} \dot{n}
$$

where the $\boldsymbol{f}_{i}, \boldsymbol{f}_{j}$ are nodal force vectors at end $i$ and $j$ of the member. It is noted that the first and second terms on the right hand side of Equation (6) correspond to geometrical stiffness and elastic stiffness, respectively.

Differentiation of Equation (2a) and then substitution of Equation (2c) into the resulting differentation will yield

$$
{ }^{t} \dot{\lambda}=\frac{1}{{ }^{t} \dot{l}+{ }^{t} \dot{u}}\left[-\left(\boldsymbol{I}_{3}-{ }^{t} \lambda^{t} \lambda^{\mathrm{T}}\right)\left(\boldsymbol{I}_{3}-{ }^{t} \lambda^{t} \lambda^{\mathrm{T}}\right)\right]\left\{\begin{array}{c}
{ }^{t} \dot{\boldsymbol{x}}_{i} \\
{ }^{t} \dot{\boldsymbol{x}}_{j}
\end{array}\right\}
$$

where $I_{3}$ is a $3 \times 3$ identity matrix. Substitution of Equation (7) into the first term on the right hand side of Equation (6) yields

$$
\left[\begin{array}{c}
-{ }^{t} \dot{\lambda} \\
{ }^{t} \dot{\lambda}
\end{array}\right]{ }^{t} n={ }^{t} \boldsymbol{k}_{g}\left\{\begin{array}{c}
{ }^{t} \dot{\boldsymbol{x}}_{i} \\
{ }^{t} \dot{\boldsymbol{x}}_{j}
\end{array}\right\}
$$

where ${ }^{t} \boldsymbol{k}_{g}$ is the $6 \times 6$ geometrical stiffness matrix which is defined as follows:

$$
{ }^{t} \boldsymbol{k}_{g}=\frac{{ }^{t-1} n}{{ }^{t-1} l+{ }^{t-1} u}\left[\begin{array}{cc}
{\left[\boldsymbol{I}_{3}-{ }^{t} \lambda^{t} \lambda^{\mathrm{T}}\right]} & -\left[\boldsymbol{I}_{3}-{ }^{t} \lambda^{t} \lambda^{\mathrm{T}}\right] \\
-\left[\boldsymbol{I}_{3}-{ }^{t} \lambda^{t} \lambda^{\mathrm{T}}\right] & {\left[\boldsymbol{I}_{3}-{ }^{t} \lambda^{t} \lambda^{\mathrm{T}}\right]}
\end{array}\right]
$$

Considering Equation (4b), the second term on the right hand side of Equation (6) could be expressed as

$$
\left\{\begin{array}{c}
-{ }^{t} \boldsymbol{\lambda} \\
{ }^{t} \boldsymbol{\lambda}
\end{array}\right\}{ }^{t} \dot{n}={ }^{t} \boldsymbol{k}_{e}\left\{\begin{array}{c}
{ }^{t} \dot{\boldsymbol{x}}_{i} \\
{ }^{t} \dot{\boldsymbol{x}}_{j}
\end{array}\right\}-\left\{\begin{array}{c}
-{ }^{t} \boldsymbol{\lambda} \\
{ }^{t} \boldsymbol{\lambda}
\end{array}\right\} \frac{E A}{{ }^{t-1} l}\left(1+\frac{{ }^{t-1} u}{{ }^{t-1} l}\right) t
$$


where ${ }^{t} \boldsymbol{k}_{e}$ is the $6 \times 6$ linear stiffness matrix which is given by the following expression:

$$
{ }^{t} \boldsymbol{k}_{e}=\frac{E A}{{ }^{t-1} l}\left[\begin{array}{cc}
{ }^{t} \lambda^{t} \lambda^{\mathrm{T}} & -{ }^{t} \lambda^{t} \lambda^{\mathrm{T}} \\
-{ }^{t} \lambda^{t} \lambda^{\mathrm{T}} & { }^{t} \lambda^{t} \lambda^{\mathrm{T}}
\end{array}\right]
$$

By assembling all element contribution for the second term on the right side of Equation (10), the following equation for the entire structure could be obtained:

$-\sum_{\text {member }}\left\{\begin{array}{c}-{ }^{t} \lambda \\ { }^{t} \boldsymbol{\lambda}\end{array}\right\} \frac{E A}{{ }^{t-1} l}\left(1+\frac{{ }^{t-1} u}{{ }^{t-1} l}\right){ }^{t} i=-{ }^{t} \boldsymbol{B}^{t} \boldsymbol{C}_{L}{ }^{t} \boldsymbol{l}$

where ${ }^{t} \boldsymbol{C}_{L}$ is a $m \times m$ matrix which could be further expressed as the summation of two $m \times m$ matrices ${ }^{t} \boldsymbol{C}_{1}$ and ${ }^{t} \boldsymbol{C}_{2}$ as follows:

$$
\begin{aligned}
& { }^{t} \boldsymbol{C}_{L}={ }^{t} \boldsymbol{C}_{1}+{ }^{t} \boldsymbol{C}_{2} \\
& { }^{t} \boldsymbol{C}_{1}=\left[\begin{array}{cccc}
\frac{E_{1} A_{1}}{{ }^{t-1} l_{1}} & & & \\
& \frac{E_{2} A_{2}}{{ }^{t-1} l_{2}} & & \\
& & \ddots & \\
& & & \frac{E_{m} A_{m}}{{ }^{t-1} l_{m}}
\end{array}\right] \\
& { }^{t} \boldsymbol{C}_{2}=\left[\begin{array}{lll}
\frac{E_{1} A_{1}}{{ }^{t-1} l_{1}\left(\frac{{ }^{t-1} u_{1}}{{ }^{t-1} l_{1}}\right)} & & \\
& \frac{E_{2} A_{2}}{{ }^{t-1} l_{2}}\left(\frac{{ }^{t-1} u_{2}}{{ }^{t-1} l_{2}}\right) & \\
& \ddots & \\
& & \frac{E_{m} A_{m}}{{ }^{t-1} l_{m}}\left(\frac{{ }^{t-1} u_{m}}{{ }^{t-1} l_{m}}\right)
\end{array}\right]
\end{aligned}
$$

By assembling all element contribution for Equation (8) and the first term on the right side of Eq.(11) and then considering Eq.(12), incremental equation (see Equation (5)) for the entire structure could be obtained as follows:

$$
\begin{aligned}
{ }^{t} \dot{\boldsymbol{F}} & ={ }^{t} \boldsymbol{K}^{t} \dot{\boldsymbol{x}}-{ }^{t} \boldsymbol{B}^{t} \boldsymbol{C}_{L}{ }^{t} \boldsymbol{i} \\
{ }^{t} \boldsymbol{K} & =\sum_{\text {member }}\left({ }^{t} \boldsymbol{k}_{e}+{ }^{t} \boldsymbol{k}_{g}\right)
\end{aligned}
$$

where ${ }^{t} \boldsymbol{K}$ is the tangent stiffness matrix at the structure level. Since the tensegrity under feasible prestress is a stable structure, the inverse of ${ }^{t} \boldsymbol{K}$ exists. Under constant-loading $\left({ }^{t} \dot{\boldsymbol{F}}=\boldsymbol{0}\right)$, Equation (14) could be solved for incremental nodal coordinates for the whole structure as follows:

$$
{ }^{t} \dot{\boldsymbol{x}}_{G}={ }^{t} \boldsymbol{K}^{-1}{ }^{t} \boldsymbol{B}^{t} \boldsymbol{C}_{\mathrm{L}}{ }^{t} \boldsymbol{i}
$$

where ${ }^{t} \dot{\boldsymbol{x}}_{\mathrm{G}}$ is the vector of incremental coordinates for the entire structure. 
Making use of Equation (15) and the corresponding form of Equation (2c) for the whole structure, we will first get:

$$
{ }^{t} \dot{\boldsymbol{u}}={ }^{T} \boldsymbol{B}^{t} \dot{\boldsymbol{x}}_{G}-{ }^{t} \boldsymbol{i}=\left({ }^{T} \boldsymbol{B}^{t} \boldsymbol{K}^{-1}{ }^{t} \boldsymbol{B}^{t} \boldsymbol{C}_{L}-\boldsymbol{I}_{m}\right)^{t} \boldsymbol{i}
$$

Upon substitution of Equation (16a) into the corresponding form of Equation (4b) for the entire structure and taking note of Equation (13), we will then arrive at the following equation for incremental axial force:

$$
\begin{gathered}
{ }^{t} \dot{\boldsymbol{n}}={ }^{t} \boldsymbol{D}_{2}{ }^{t} \boldsymbol{i} \\
{ }^{t} \boldsymbol{D}_{2}=\left({ }^{t} \boldsymbol{C}_{1}{ }^{t} \boldsymbol{B}^{T}{ }^{t} \boldsymbol{K}^{-1}{ }^{t} \boldsymbol{B}-\boldsymbol{I}_{m}\right){ }^{t} \boldsymbol{C}_{L}
\end{gathered}
$$

\subsection{Optimization of forced elongation}

During shape change analysis, the advancement of the monitored nodes to approach the target coordinates is achieved by minimizing the following objective function:

$\min f(\boldsymbol{x})=\left|{ }^{t} \boldsymbol{x}^{\prime}-\overline{\boldsymbol{x}}\right|$

where $\overline{\boldsymbol{x}}$ is prescribed target coordinates, and ${ }^{t} \boldsymbol{x}^{\prime}={ }^{t-1} \boldsymbol{x}^{\prime}+{ }^{t} \dot{\boldsymbol{x}}^{\prime}$ is the current coordinates, for all the specified monitored nodes at current step $t$ during the shape change analysis.

The incremental nodal coordinates for the specified monitored nodes, ${ }^{t} \dot{\boldsymbol{x}}^{\prime}$, are extracted from the incremental nodal coordinates of all nodes in a tensegrity, ${ }^{t} \dot{\boldsymbol{x}}$, (see Equation (15)) by introducing matrix $\boldsymbol{B}_{1}$ as follows:

${ }^{t} \dot{\boldsymbol{x}}^{\prime}=\boldsymbol{B}_{1}{ }^{t} \dot{\boldsymbol{x}}$

where matrix $\boldsymbol{B}_{1}$ consists of only value 1 or 0 . It contains information about the prescribed target coordinates of the monitored nodes. Size of matrix $\boldsymbol{B}_{1}$ is $n_{u t g} \times n_{u}$, where $n_{u t g}$ and $n_{u}$ denote number of target coordinates and number of unconstrained degree of freedom, respectively.

By substituting Equation (15) into Equation (18), the following equation could be obtained:

$$
\begin{aligned}
{ }^{t} \dot{\boldsymbol{x}}^{\prime} & =\boldsymbol{B}_{2}{ }^{t} \boldsymbol{i} \\
\boldsymbol{B}_{2} & =\boldsymbol{B}_{1}\left({ }^{t} \boldsymbol{K}^{-1 t} \boldsymbol{B}^{t} \boldsymbol{C}_{L}\right)
\end{aligned}
$$

where $\boldsymbol{B}_{2}$ is a matrix of size $n_{u t g} \times m_{c f}$ where $m_{c f}$ is number of cables excluding the undeformed cables.

Sequential Quadratic Programming (SQP) method is employed to solve the nonlinear optimization problem as shown in Equation (17). The nonlinear problem is later approximated quadratically to formulate the following quadratic programming sub-problem:

$$
f(\boldsymbol{x})=\frac{1}{2}\left[\left({ }^{t} \boldsymbol{x}^{\prime}-\overline{\boldsymbol{x}}\right)^{\mathrm{T}}\left({ }^{t} \boldsymbol{x}^{\prime}-\overline{\boldsymbol{x}}\right)\right]
$$

which could be rewritten as follows: 
$\min _{x \in R^{n}} f(\boldsymbol{x})=\boldsymbol{g}^{T}{ }^{t} \boldsymbol{i}+\frac{1}{2}^{t} \boldsymbol{i}^{\mathrm{T}} \boldsymbol{H}^{t} \boldsymbol{i}$

subject to $\boldsymbol{A}_{2}{ }^{t} \boldsymbol{i} \geq \boldsymbol{b}_{2}$

where

$\boldsymbol{g}=\boldsymbol{B}_{2}{ }^{\mathrm{T}}\left({ }^{t-1} \boldsymbol{x}^{\prime}-\overline{\boldsymbol{x}}\right)$

$\boldsymbol{H}=\boldsymbol{B}_{2}{ }^{\mathrm{T}} \boldsymbol{B}_{2}$

${ }^{t} \boldsymbol{i}$ is a $m_{c f} \times 1$ vector of incremental forced elongation which corresponds to the optimization variable and $\boldsymbol{H}$ is a positive-definite approximation of Hessian matrix of the Lagrangian function. Contents of matrix $\boldsymbol{A}_{2}$ and vector $\boldsymbol{b}_{2}$ appearing in the inequality constraint equation are further explained below.

In this study, the SQP problem as represented by Equation (21) is subjected to inequality constraints, corresponding to the limitation in allowable axial forces and forced elongation. The first constraint on axial forces could be expressed as

$$
\boldsymbol{n}_{L}-{ }^{t-1} \boldsymbol{n} \leq \boldsymbol{D}_{2}{ }^{t} \boldsymbol{i} \leq \boldsymbol{n}_{U}-{ }^{t-1} \boldsymbol{n}
$$

where $\boldsymbol{n}_{L},{ }^{t-1} \boldsymbol{n}, \boldsymbol{D}_{2}{ }^{t} \boldsymbol{i}, \boldsymbol{n}_{U}$ are vectors of lower limit of axial force, axial force at previous step, incremental axial force (see Equation (16)) and upper limit of axial force, respectively. For elastic material properties, the lower and upper axial force limit for cables and struts could be defined as:

$$
0 \leq n_{c} \leq \sigma_{c} A_{c}
$$

$$
-\frac{\pi^{2} E_{s} I_{s}}{l_{s}^{2}} \leq n_{s} \leq \sigma_{s} A_{s}
$$

where $n_{c}, \sigma_{c}$ and $A_{c}$ are axial forces, yield stress and cross sectional area for cable elements, respectively; $n_{s}, E_{s}, l_{s}, L_{s}, \sigma_{s}$ and $A_{s}$ are axial forces, Young modulus, moment of inertia, element current length, yield stress and cross sectional area for strut elements, respectively. In this study, all strut elements have circular cross sectional area. As shown in Equation (23), in addition to checking for yielding in cable elements, compressive forces of strut elements are checked against Euler's buckling load.

A limit of $d_{c l}$ (with $d_{c l}>0$ ) which is imposed on each variable forced elongation ' $i$ is taken as the second constraint which is expressed as

$$
-d_{c l} \leq{ }^{t} i \leq d_{c l}
$$

or

$$
-\boldsymbol{e}_{c f} \leq \boldsymbol{I}^{t} \boldsymbol{i} \leq \boldsymbol{e}_{c f}
$$

for all $m_{c f}$ cables subjected to forced elongation where $\boldsymbol{e}_{c f}$ is a $m_{c f} \times 1$ of which the entries are all $d_{c l}$,

$I_{m c f}$ is a $m_{c f} \times m_{c f}$ identity matrix and $m_{c f}$ is the number of cables excluding the undeformed cables. Negative and positive values in $d_{c l}$ indicates shortening and lengthening of cable in tensegrity, respectively.

By using Equations (22) and (24), matrix $\boldsymbol{A}_{2}$ and $\boldsymbol{b}_{2}$ in Equation (21) could be written in matrix form as 


$$
\boldsymbol{A}_{2}=\left\{\begin{array}{c}
\boldsymbol{D}_{2} \\
\cdots \\
-\boldsymbol{D}_{2} \\
\cdots \\
\boldsymbol{I}_{m c f} \\
\cdots \\
-\boldsymbol{I}_{m c f}
\end{array}\right\} ; \boldsymbol{b}_{2}=\left\{\begin{array}{c}
\boldsymbol{n}_{L}-{ }^{t-1} \boldsymbol{n} \\
\cdots \\
-\left(\boldsymbol{n}_{U}-{ }^{t-1} \boldsymbol{n}\right) \\
\cdots \\
-d_{c l} \\
\cdots \\
-d_{c l}
\end{array}\right\}
$$

where $\boldsymbol{A}_{2}$ is a $\left(2 m+2 m_{c f}\right) \times m_{c f}$ matrix and $\boldsymbol{b}_{2}$ is a $\left(2 m+2 m_{c f}\right) \times 1$ vector.

It is noted SQP method determines ${ }^{t} \boldsymbol{i}$ by solving the quadratic programming subproblem iteratively. Assuming that the given approximation for actual solution ${ }^{t} \boldsymbol{i}^{m}$ is applied to the subproblem at $m^{\text {th }}$ iteration, the new iterate ${ }^{t} \boldsymbol{l}^{m+1}$ is obtained as follows:

$$
{ }^{t} \boldsymbol{i}^{m+1}={ }^{t} \boldsymbol{i}^{m}+{ }^{t} \boldsymbol{i}
$$

where ${ }^{t} \boldsymbol{i}$ is the solution to the subproblem. The iteration is repeated until the $\left({ }^{t} \boldsymbol{l}^{m}\right)_{m \in R^{n}}$ converges to the local minimum, ${ }^{t} \boldsymbol{i} *$. The obtained converged ${ }^{t} \boldsymbol{i}$ is then used in each incremental step of shape change analysis to determine the updated incremental nodal coordinates and incremental axial forces.

\section{PROPOSED ALGORITHM FOR SHAPE CHANGE ANALYSIS}

Figure 6 shows the proposed algorithm for shape change analysis of tensegrity. Algorithm 1 is the main algorithm showing the computational strategies for shape change analysis of tensegrity model. Algorithm 2 is an independent algorithm for searching the optimized forced elongation.

\section{Algorithm 1 (Shape change analysis)}

i. Step 1: Preparation of initial state information for tensegrity Set $t=0$ for the initial state of analysis. At this step, the topology of the tensegrity is defined to satisfy being statically and kinematically indeterminate. In addition, the existence of tensile self-stress in cables is necessary for the tensegrity at the initial state before proceeding to the following stage. The axial forces at the initial selfequilibrium state are determined from the homogenous equation $\mathbf{B n}=\mathbf{0}$ in Equation (1). Basically, the linearly independent self-equilirium mode is determined by using the generalized inverse method (Hangai and Kawaguchi, 1991; Oh et al., 2016). Furthermore, a minimization procedure was used to determine a possible combination of coefficients of linearly independent self-equilibrium modes subjected to proper constraints on forces in cable and struts (Oh, 2017).

ii. Step 2: Preparation of deformed state information for tensegrity Set $t=1$. In the deformed state, external loads and boundary conditions are applied to the tensegrity. In this study, external load corresponding to the self-weight is applied to all nodes in the tensegrity. The three nodes forming the triangular surface at the base are fixed. Upon loading, the nodal coordinates and axial forces of the tensegrity is updated through Equation (3).

iii. Step 3: Specification of monitored nodes and target displacements Set $t=2$ for the starting step of shape change state. Specification of monitored nodes and their target coordinates is important for the optimization of forced elongation in Algorithm 2. In this study, three nodes at the top of tensegrity are chosen as the monitored nodes. The corresponding target coordinates are discussed later in Section 4.0.

iv. Step 4: Algorithm 2 (Optimization of cables forced elongation) Algorithm 2 aims to determine the optimized forced elongation for cables. In Algorithm 2, SQP method is employed in the optimization problem. The forced elongation for cable is determined by solving the quadratic programming sub-problem subjected to inequality constraints as shown in Equation (21). 
v. Step 5: Preparation of shape change state information for tensegrity

Once the optimized forced elongation of cables is determined, the incremental nodal coordinates as shown in Equation (15) and incremental axial forces as shown in Equation (16) are solved. This leads to the determination of current axial forces and current nodal coordinates at step $t=i$ in shape change state.

vi. Step 6: Calculation of objective function Objective function as shown in Equation (17) is calculated.

vii. Step 7: Checking for satisfaction of termination criteria Analysis in Algorithm 1 is checked against either of the following two termination criteria: the objective function less than 0.1 or the maximum iteration greater than 10000 . If either one of these criteria is satisfied, Algorithm 1 is terminated; otherwise step $t=i+1$ is set and Algorithm 1 is repeated by determination of new set of forced elongation using Algorithm 2 (see Figure 6).

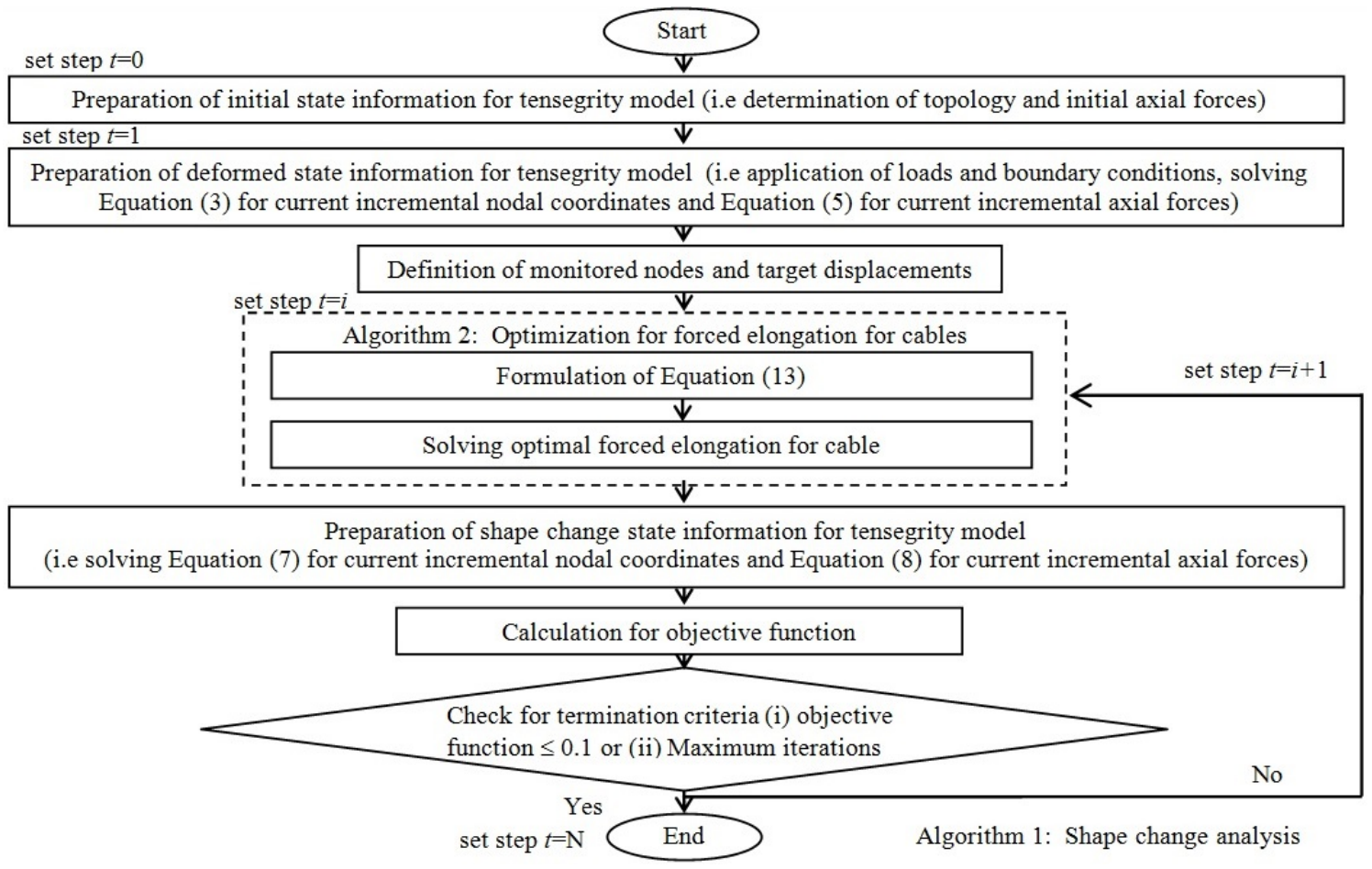

Figure 6: Algorithm for shape change analysis.

\section{NUMERICAL EXAMPLES}

In this section, four models of tensegrities are used as numerical examples to illustrate the applicability of the proposed approach for shape change analysis. All four tensegrities are assigned the same boundary and loading conditions. They are different in terms of size, method of assembly and prestresses pattern.

\subsection{Topology characterization}

The proposed algorithm for shape change analysis is applied to the following four models of tensegrities: simplex, quadruplex, two-stage tensegrity and tapered three-stage tensegrity models. Figure 7 shows the geometrical (i.e. nodal coordinates and member connectivity) of the studied tensegrity models. The struts and cables of tensegrity models are denoted using circular tubes and lines, respectively. 


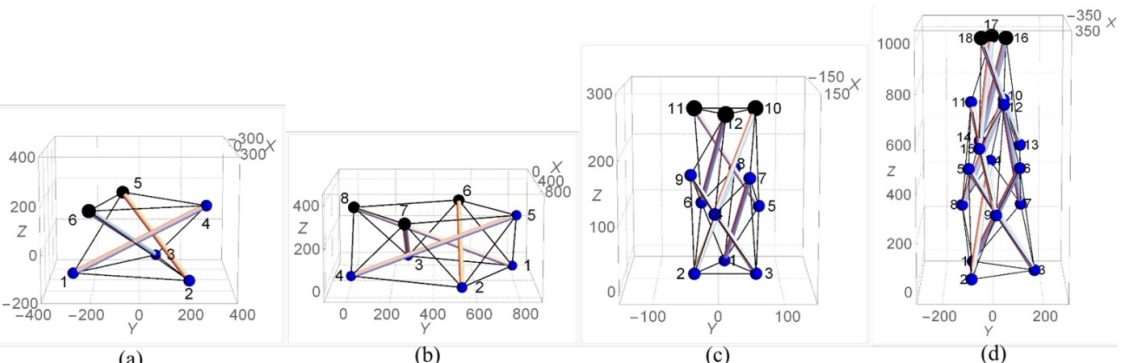

Figure 7: Tensegrity models (a) simplex, (b) quadruplex, (c) two-stage and (d) tapered three-stage. (n.t.s)

The first model is a simplex (Figure 7a) of three struts and nine cables with a relative twist angle of $30^{\circ}$ between the upper and lower triangular surfaces. The second model, a quadruplex (Figure 7b) is generated based on an individually reinforced quadruplex unit of a clustered tensegrity structure investigated by Ali et al. (2011). The quadruplex consists of four struts and sixteen cables. The third model is a two-stage tensegrity model (Figure 7c) which is slightly modified from the topology examined by Pinaud (2005) by adding three reinforcing cables. The model composes of six struts and twenty seven cables. The forth model, a tapered tensegrity model (Figure 7d) with aspect ratio (width/height) of $1: 3.3$ is modeled with the base width and top width measuring $300 \mathrm{~mm}$ and $100 \mathrm{~mm}$, respectively. The overall model consists of a stack of three independent simplex units with nominal height of $450 \mathrm{~mm}$ each. The upper unit sinks into the lower unit with constant sink depth of $180 \mathrm{~mm}$. Besides, the upper and lower equilateral triangular surfaces of each simplex unit are twisted with relative angle of $25^{\circ}$. The triangular sides are $300 \mathrm{~mm}$ and $100 \mathrm{~mm}$ at the base and top of the model, respectively whereas the triangular sides for those intermediate triangles between the base and top of the tapered tensegrity model are scaled proportional to the ratio of the height of the triangle to the total height of the model. The overall model consists of nine struts and forty two cables.

As mentioned earlier, all tensegrity models are subjected to only selfweight, and all displacements of the nodes at the base are constrained. Table 1-3 are material properties, member connectivity, and nodal coordinates for all the models.

Table 1: Material Properties.

\begin{tabular}{|c|c|c|c|c|c|}
\hline & $\begin{array}{l}\text { Elastic Modulus, } E \\
\text { (MPa) }\end{array}$ & $\begin{array}{l}\text { Yield Stress, } \sigma_{\mathrm{s}} \\
\quad(\mathrm{MPa})\end{array}$ & $\begin{array}{l}\text { Density, } v \\
\left(\mathrm{~kg} / \mathrm{mm}^{3}\right)\end{array}$ & $\begin{array}{l}\text { Strut Cross sectional } \\
\quad \text { Area, } A_{\mathrm{s}}\left(\mathrm{mm}^{2}\right)\end{array}$ & $\begin{array}{c}\text { Cable Cross sectional } \\
\text { Area, } A_{c}\left(\mathrm{~mm}^{2}\right)\end{array}$ \\
\hline Simplex & 200000 & 250 & $7.85 \times 10^{6}$ & 530.90 & 50.27 \\
\hline Quadruplex & & & & 201.00 & 52.60 \\
\hline Two-stage & & & & 50.27 & 0.79 \\
\hline Tapered three-stage & & & & 113.10 & 19.63 \\
\hline
\end{tabular}

Table 2: Member connectivity.

\begin{tabular}{|c|c|c|c|c|c|c|c|c|c|c|}
\hline \multicolumn{3}{|c|}{ Members } & \multirow{2}{*}{$\frac{\text { Simplex }}{* 1,4,1}$} & \multicolumn{2}{|c|}{ Quadruplex } & \multicolumn{2}{|c|}{ Two-stage } & \multicolumn{3}{|c|}{ Tapered three-stage } \\
\hline 1 & 18 & 35 & & $1,8,1$ & $2,7,2$ & $1,7,1$ & $7,5,2$ & $1,6,1$ & $5,9,2$ & $8,11,2$ \\
\hline 2 & 19 & 36 & $2,5,1$ & $2,6,1$ & $3,6,2$ & $2,8,1$ & $3,7,2$ & $2,4,1$ & $9,6,2$ & $9,12,2$ \\
\hline 3 & 20 & 37 & $3,6,1$ & $3,7,1$ & $4,8,2$ & $3,9,1$ & $2,9,2$ & $3,5,1$ & $6,7,2$ & $7,13,2$ \\
\hline 4 & 21 & 38 & $4,5,2$ & $4,5,1$ & & $4,10,1$ & $1,8,2$ & $7,12,1$ & $7,4,2$ & $8,14,2$ \\
\hline 5 & 22 & 39 & $5,6,2$ & $1,3,2$ & & $5,11,1$ & $3,4,2$ & $8,10,1$ & $10,14,2$ & $9,15,2$ \\
\hline 6 & 23 & 40 & $6,4,2$ & $3,4,2$ & & $6,12,1$ & $2,6,2$ & $9,11,1$ & $14,11,2$ & $4,10,2$ \\
\hline 7 & 24 & 41 & $1,2,2$ & $4,2,2$ & & $1,2,2$ & $1,5,2$ & $13,18,1$ & $11,15,2$ & $5,11,2$ \\
\hline 8 & 25 & 42 & $2,3,2$ & $2,1,2$ & & $2,3,2$ & $10,5,2$ & $14,16,1$ & $15,12,2$ & $6,12,2$ \\
\hline 9 & 26 & 43 & $3,1,2$ & $5,6,2$ & & $3,1,2$ & $11,6,2$ & $15,17,1$ & $12,13,2$ & $13,16,2$ \\
\hline 10 & 27 & 44 & $1,5,2$ & $6,8,2$ & & $10,11,2$ & $12,4,2$ & $1,2,2$ & $13,10,2$ & $14,17,2$ \\
\hline 11 & 28 & 45 & $2,6,2$ & $8,7,2$ & & $11,12,2$ & $10,7,2$ & $2,3,2$ & $1,4,2$ & $15,18,2$ \\
\hline 12 & 29 & 46 & $3,4,2$ & $7,5,2$ & & $12,10,2$ & $11,8,2$ & $3,1,2$ & $2,5,2$ & $10,16,2$ \\
\hline 13 & 30 & 47 & & $1,6,2$ & & $5,8,2$ & $12,9,2$ & $16,17,2$ & $3,6,2$ & $11,17,2$ \\
\hline 14 & 31 & 48 & & $3,8,2$ & & $8,6,2$ & $1,6,2$ & $17,18,2$ & $1,7,2$ & $12,18,2$ \\
\hline 15 & 32 & 49 & & $4,7,2$ & & $6,9,2$ & $2,4,2$ & $18,16,2$ & $2,8,2$ & $1,8,2$ \\
\hline 16 & 33 & 50 & & $2,5,2$ & & $9,4,2$ & $3,5,2$ & $4,8,2$ & $3,9,2$ & $2,9,2$ \\
\hline 17 & 34 & 51 & & $1,5,2$ & & $4,7,2$ & & $8,5,2$ & $7,10,2$ & $3,7,2$ \\
\hline
\end{tabular}

*Note: Information of $(1,4,1)$ in member 1 of simplex denotes as node $i$, node $j$ and structural type (type 1 is strut whereas type 2 is cable) 
Table 3: Nodal Coordinates.

\begin{tabular}{cccccc}
\hline \hline Node & Simplex & Node & Two-stage & Node & Tapered three-stage \\
\hline 1 & $77.64,-289.77,-150$ & 1 & $-42.72,9.98,0$ & 1 & $-150.00,-86.60,0$ \\
2 & $212.13,212.13,-150$ & 2 & $43.43,-38.82,0$ & 2 & $150.00,-86.60,0$ \\
3 & $-289.77,77.64,-150$ & 3 & $42.62,60.22,0$ & 3 & $0.00,173.21,0$ \\
4 & $77.64,289.77,150$ & 4 & $68.51,-5.91,102.57$ & 4 & $-120.26,-10.52,450$ \\
5 & $-289.77,-77.64,150$ & 5 & $1.61,65.45,102.57$ & 5 & $69.24,-98.89,450$ \\
6 & $212.13,-212.13,150$ & 6 & $-26.77,-28.16,102.57$ & 6 & $51.02,109.41,450$ \\
& & 7 & $55.65,49.06,157.80$ & 7 & $-81.28,116.08,270$ \\
Node & Quadruplex & 8 & $-39.57,26.84,157.80$ & 8 & $-59.89,-128.43,270$ \\
1 & $266.67,800,0$ & 9 & $27.28,-44.51,157.80$ & 9 & $141.17,12.35,270$ \\
2 & $800,533.34,0$ & 10 & $-14.60,59.66,260.37$ & 10 & $-77.28,44.61,720$ \\
3 & $0,266.67,0$ & 11 & $-13.67,-39.32,260.37$ & 11 & $0.00,-89.23,720$ \\
4 & $533.34,0,0$ & 12 & $71.60,10.99,260.37$ & 12 & $77.28,44.61,720$ \\
5 & $533.34,800,300$ & & & 13 & $0.00,110.22,540$ \\
6 & $0,533.34,300$ & & & 14 & $-95.45,-55.11,540$ \\
7 & $800,266.67,300$ & & & 15 & $95.45,-55.11,540$ \\
8 & $266.67,0,300$ & & & 16 & $24.40,52.33,990$ \\
& & & & 17 & $-57.51,-5.04,990$ \\
\end{tabular}

\subsection{Convergence results}

This section examines the computational efficiency by examining the convergence results obtained using the proposed algorithm for shape change analysis. The results reveal the effectiveness of the algorithm for a tensegrity model to achieve a prescribed set of target coordinates.

The shape change analysis cases with target displacements in uni-directional mode are studied. For the uni-directional mode cases, target coordinates are prescribed only for a single degree of freedom (either $x, y$ or $z$ ) of the monitored nodes. The analysis series under the case of target displacements in uni-directional mode are denoted as $X, Y$ and $Z$ series, respectively. Under each series, the following cases have been examined Series X (Xn, Xp), Series $Y(Y n, Y p)$ and Series(Zn, Zp). The capital letter for the cases under each series denotes the corresponding degree of freedom where the displacement is prescribed and the lower case letters $n$ (or $p$ ) denote negative (or positive) direction of the prescribed displacement.

In this paper, only the results of cases Zp, Zn and Series $Z$ and case $Y p$ in Series $Y$ are presented. Series $X p, X n$ and $\mathrm{Yn}$ are found to show similar results as series $\mathrm{Yp}$ due to symmetrical geometry of all the models. Cases $\mathrm{Zp}, \mathrm{Zn}$ and $\mathrm{Yp}$ represent cases of tensegrity models undergoing specifically axial extensional, axial shortening and sideway deformation, respectively. Here, all the top nodes are chosen as monitored nodes. Magnitude of the target displacements for the monitored nodes is specified as half height of the tensegrity models. It is noted that there are top nodes which are unable to reach the specific target coordinates of monitored nodes owing to the limitation of strut length. For instance, node 4 and 5 for simplex (see Figure 7a) and quadruplex (see Figure 7b), respectively in case Yp are therefore not considered as monitored nodes.

Table 4 shows the total computational step for the shape change analysis of tensegrity models. The results show that higher total computational steps is always demonstrated in shape change analysis in series Zp. Beside, higher total computational step is also recorded in multiple-stage tensegrity models in case Zn compared to case Yp. Nevertheless, the opposite trend is observed in single-stage tensegrity models (simplex and quadruplex).

Since linearized incremental analysis procedure is adopted for shape change analysis without iteration step, the limit on the magnitude of forced elongation of cable $d_{c l}$ has been set to be 0.5 (see Equation 24). Such relatively small limit of $d_{c l}(0.096 \%-0.148 \%, 0.084 \%-0.125 \%, 0.315 \%-0.633 \%, 0.109 \%-0.500 \%$ of cable length, respectively for simplex, quadruplex, two-stage and tapered tensegrities) was chosen to ensure accuracy of analysis during each incremental step. The relatively small limiting value of forced elongation of cable resulted in larger total computational steps in cases with larger target displacement magnitudes specifically in cases Zn and Zp. This explains larger total computational steps in tapered three-stage tensegrity model compared to other tensegrity models with lower prescribed target displacement magnitude. This also shows that the forced elongations of cables have direct 
relationship to the displacement direction of the model only in cases $\mathrm{Zn}$ and $\mathrm{Zp}$ where the models demonstrated axial shortening and axial elongation during the shape change process.

Table 4: Total computational step in cases of Zp, Zn and Yp.

\begin{tabular}{ccccc}
\hline \hline Series & Simplex & Quadruplex & Two-stage & Tapered three-stage \\
\hline $\mathrm{Zp}$ & 195 & 114 & 74 & 227 \\
$\mathrm{Zn}$ & 70 & 70 & 48 & 119 \\
$\mathrm{Yp}$ & 91 & 189 & 22 & 34 \\
\hline \hline
\end{tabular}

Figure 8 shows plots of the normalized objective function (NOF) over computational steps for the cases of tensegrity models in cases Zp, Zn and Yp. All NOF curves clearly show the convergence at the end of the computational step. Similar decreasing trends are observed in the NOF curves within the same case.

For instance, all NOF curves clearly demonstrate the linear convergence trend in case Zp. In cases of Zn and Yp, nonlinear convergence trend of the curves is observed. In addition, faster convergence rate is clearly exhibited in the NOF curves of multi-stage tensegrity models (i.e. two-stage and tapered three-stage) particularly in case Yp.

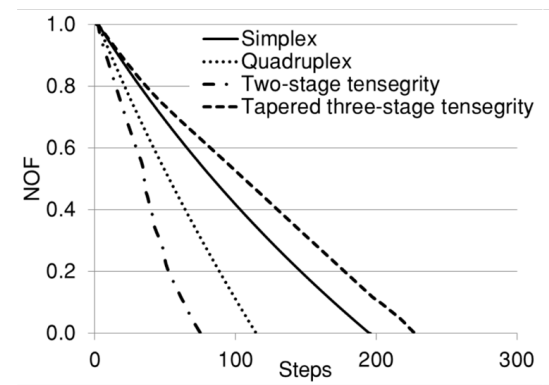

(a)

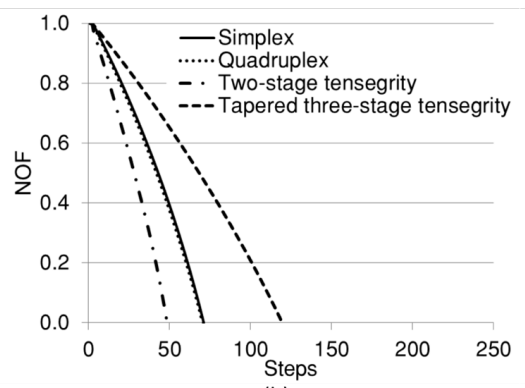

(b)

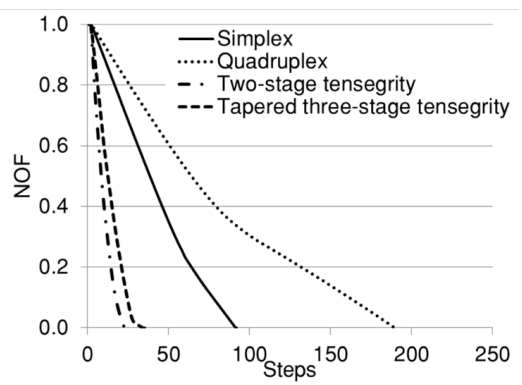

(c)

Figure 8: Normalized objective function plots for cases of (a) Zp, (b) Zn and (c) Yp.

\subsection{Shape change configurations}

Configurations of simplex, quadruplex, two-stage and tapered three-stage tensegrity model during the shape change analysis cases for series $\mathrm{Zp}, \mathrm{Zn}$ and $\mathrm{Yp}$ are presented and discussed in this section. All monitored nodes of tensegrity models have successfully approached to the target coordinates in series Zp, Zn and Yp.

\section{Case Zp}

Figure 9a and Figure 9b show the shape change of simplex and quadruplex, respectively. Both models show constant increase in height over the computational steps. Constant reduction in length for all the top horizontal cables and extension in length for the diagonal cables are also observed.

Figure 9c and Figure 9d show the shape change of two-stage and tapered three-stage tensegrity models, respectively. Along with the increment in height, these models show gradual contraction at the interfaces between two independent units and at the top surface. The sag depth for the interfaces decreases throughout the analysis. Besides, obvious twisting is observed particularly in tapered three-stage tensegrity model at the beginning of the shape change analysis.

\section{Case $\mathrm{Zn}$}

In case $\mathrm{Zn}$, all the monitored nodes for simplex (Figure 10a) and quadruplex (Figure 10b) displace downward steadily throughout the shape change analysis. Constant twisting accompanied by gradual extension in top horizontal cables is demonstrated in both models. Specifically, obvious rotation in counterclockwise direction and expansion of the top triangular surface are observed in simplex. Only slight rotation in clockwise direction is observed for quadruplex.

Two-stage tensegrity model (Figure 10c) and tapered tensegrity model (Figure 10d) displace downward with obvious expansion at the interfaces of two independent units. Twisting of the model in either counterclockwise or clockwise direction is also observed during the shape change analysis. Even though with lesser magnitude of rotation, participation of far-away nodes (such as node 7, 8 and 9) in the twisting effect is detected. 

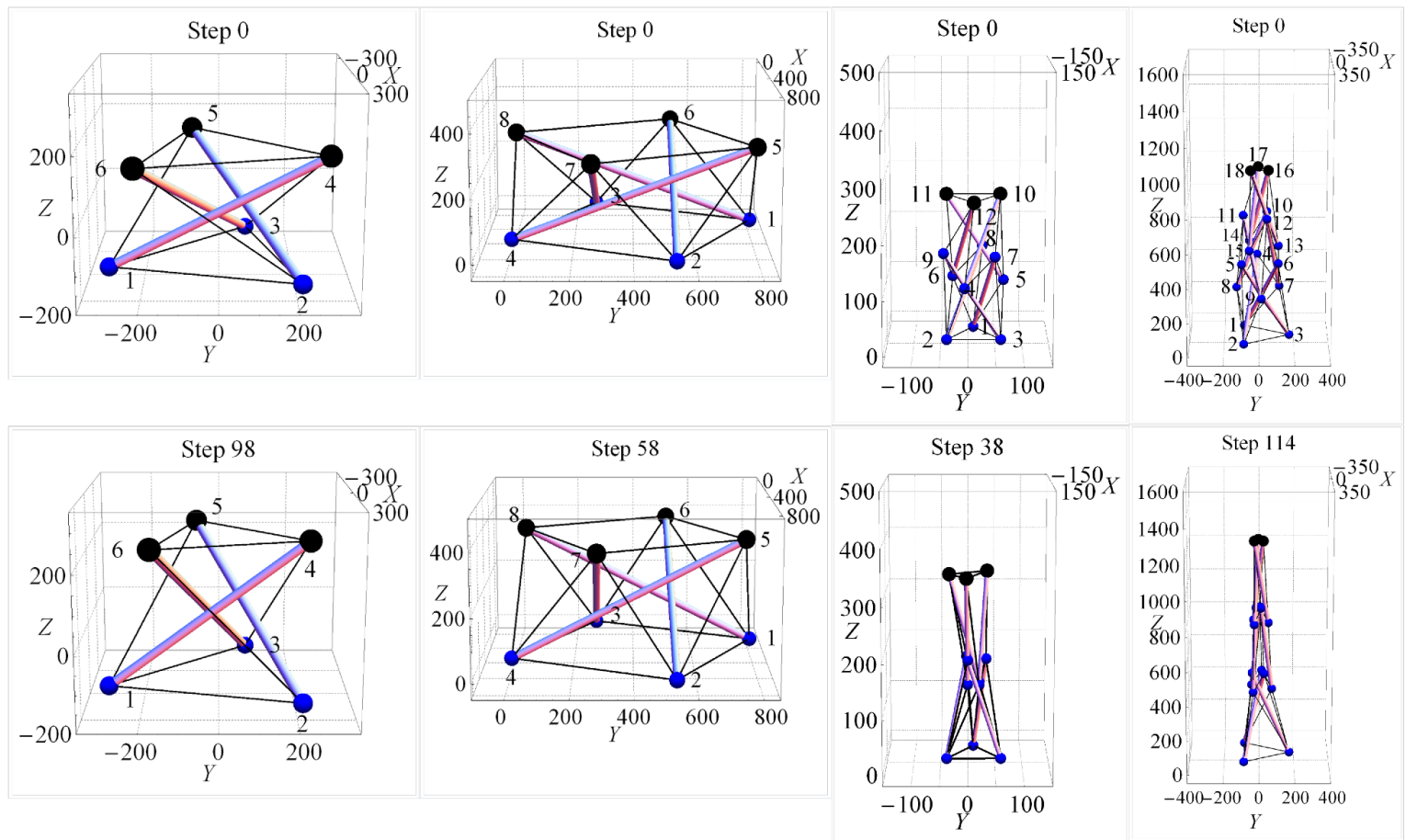

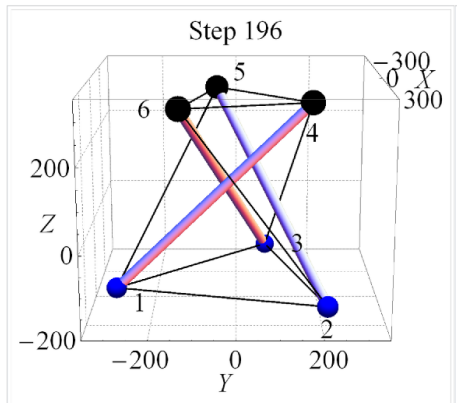

(a)

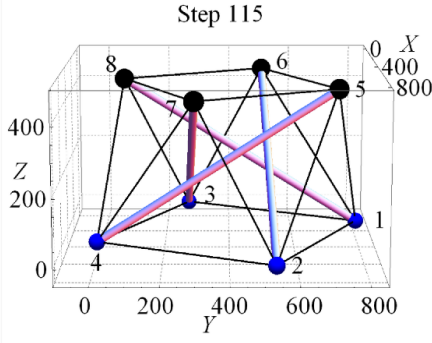

(b)

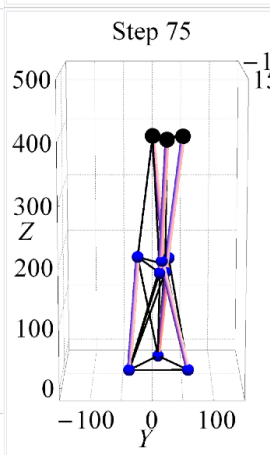

(c)

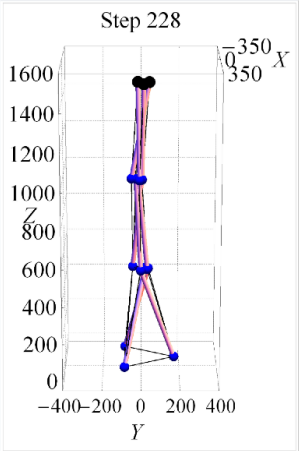

(d)

Figure 9: Shape change of tensegrity models for case Zp (a) simplex, (b) quadruplex, (c) two-stage and (d) tapered three-stage. 

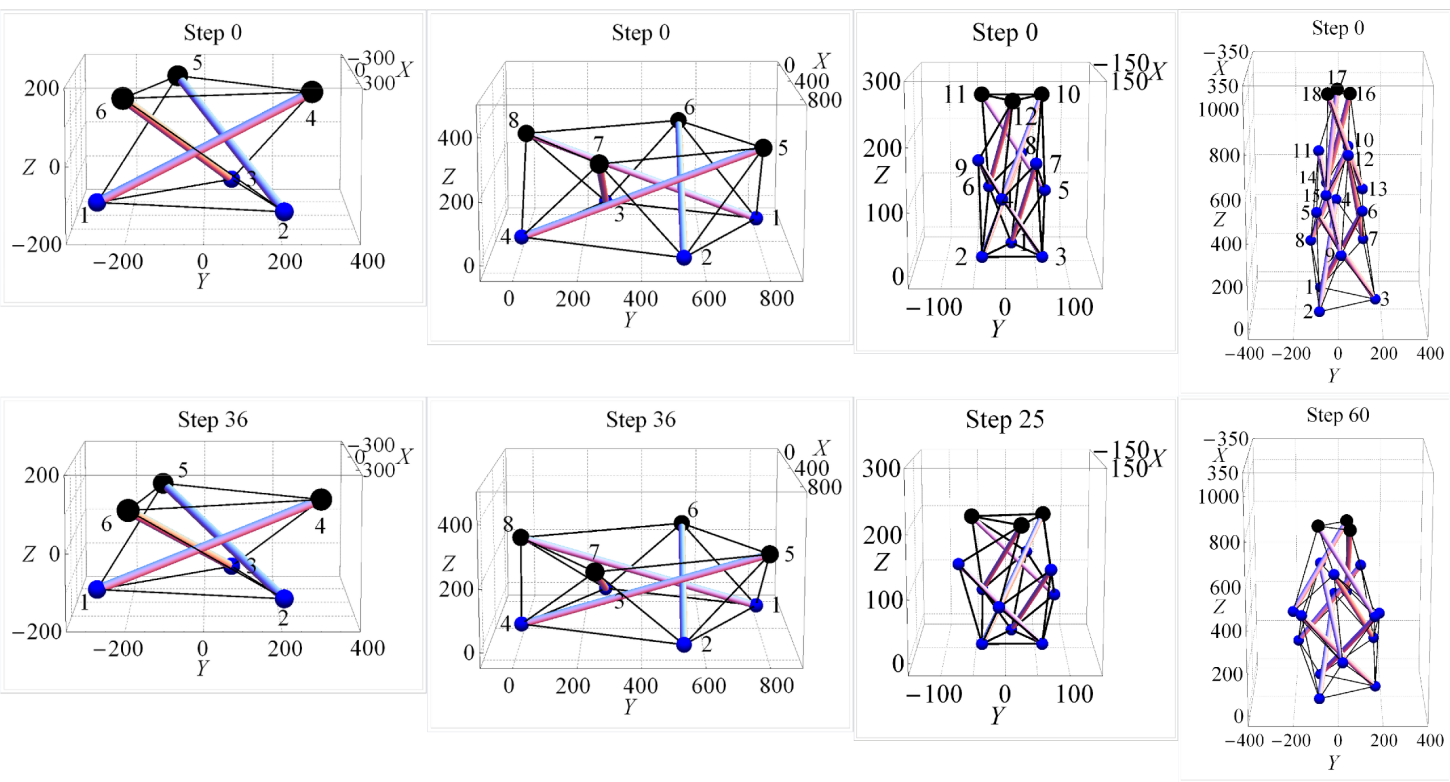

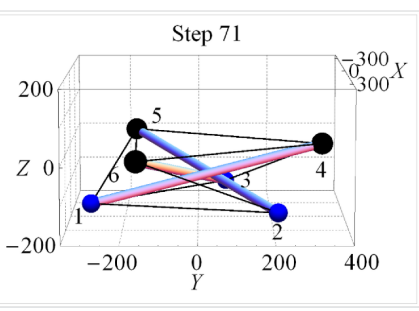

(a)

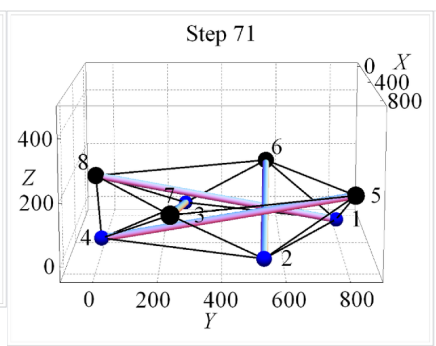

(b)

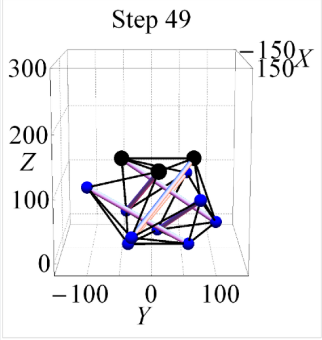

(c)

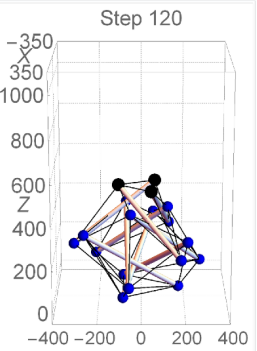

(d)

Figure 10: Shape change of tensegrity models for case Zn (a) simplex, (b) quadruplex, (c) two-stage and (d) tapered three-stage.

Case Yp

In case $\mathrm{Yp}$, all the tensegrity models displace towards positive y direction and resemble a loaded cantilever beam. Specifically monitored nodes in simplex (i.e. node 5 and 6) increase in height while the remaining top node (i.e. node 4) displaces downward simultaneously (Figure 11a). Likewise, in quadruplex (Figure 11b), node 5 constantly displaces downward whereas the height of the rest of the top nodes (i.e node 6, 7 and 8 ) is found to increase. No obvious twist is observed in both models during the shape change analysis.

Among the monitored nodes in the two-stage tensegrity model (Figure 11c), node 10 and 12 displace upward whereas node 11 displaces downward in the process of achieving target coordinates of monitored nodes. Apparent height decrement in node 5 and height increment in node 6 may further explain this situation. However unlike the rest of the nodes at the interface, node 6 does not displace towards positive y direction.

On the other hand, the tapered three-stage tensegrity model (Figure 11d) experiences bending deformation that is accompanied by displacement in y direction. All the monitored nodes of tapered three-stage tensegrity model continuously displace downward. Overall, the monitored nodes of the model show total downward displacement of $10-19 \%$ of height of model.

Figure 12 shows the length of the element for all tensegrity models at step 1 and final computational step. Similar trend was observed where the models behave like cantilever beam with the cables at the tension side tend to elongate and the one at the compression side tend to shorten. Specifically, cables 7 in Simplex, cable 20 in Quadruplex, cable 15 in Two-stage and cable 49 in Tapered three-stage presents the positive elongation of $81 \mathrm{~mm}, 187 \mathrm{~mm}, 20 \mathrm{~mm}$ and $32 \mathrm{~mm}$, respectively. On the other hand, cables 8 in Simplex, cable 17 in Quadruplex, cable 27 in Two-stage and cable 30 in Tapered three-stage presents the negative elongation of $-81 \mathrm{~mm},-186 \mathrm{~mm},-20 \mathrm{~mm}$ and $-32 \mathrm{~mm}$, respectively. The multi-stage tensegrity models elongate the least to achieve their target coordinates and reflected from the total computational step. 

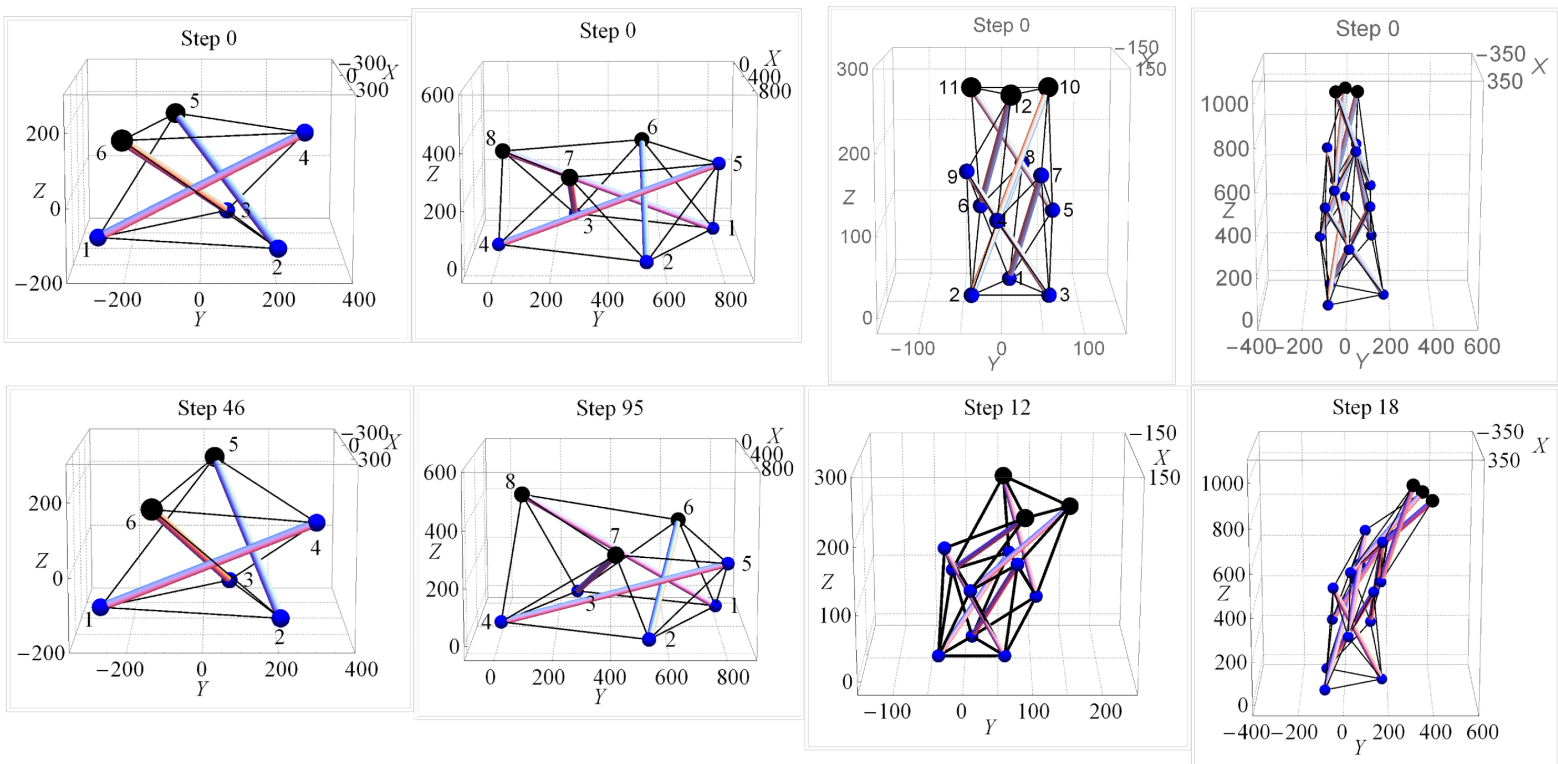

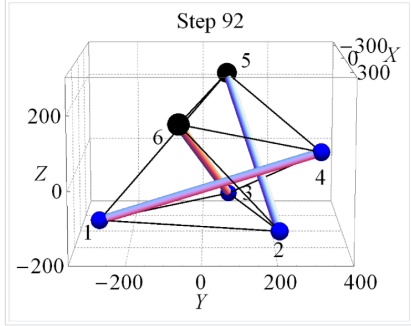

(a)

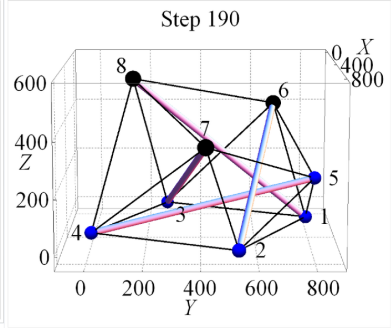

(b)

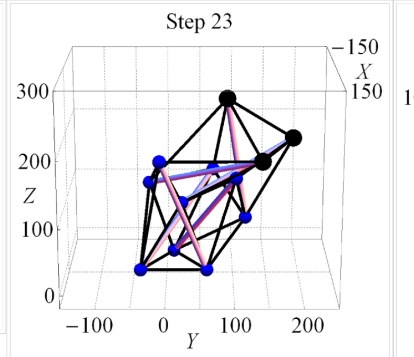

(c)

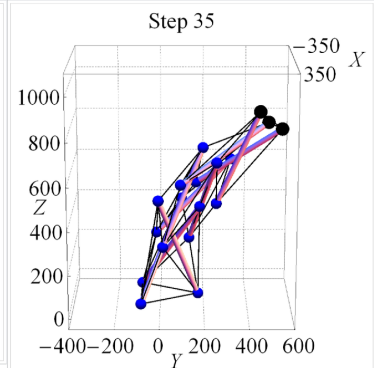

(d)

Figure 11: Shape change of tensegrity models for case Yp (a) simplex, (b) quadruplex, (c) two-stage and (d) tapered three-stage.
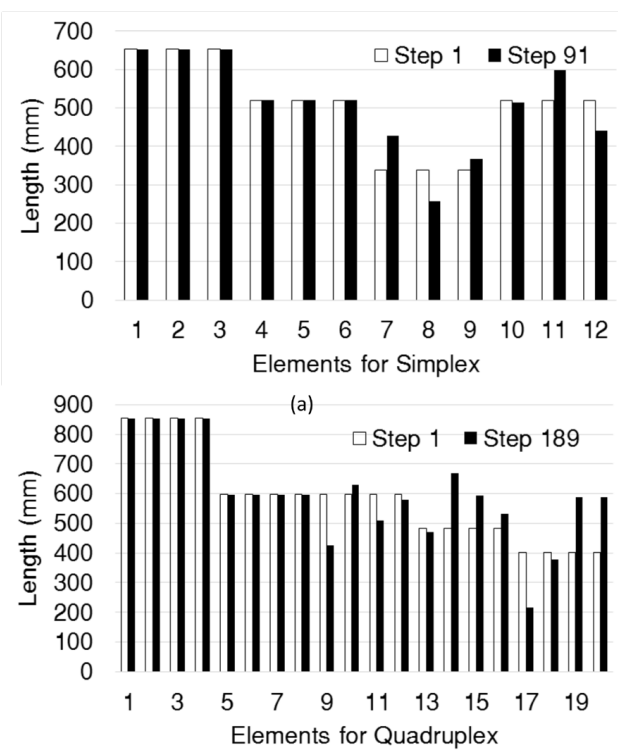
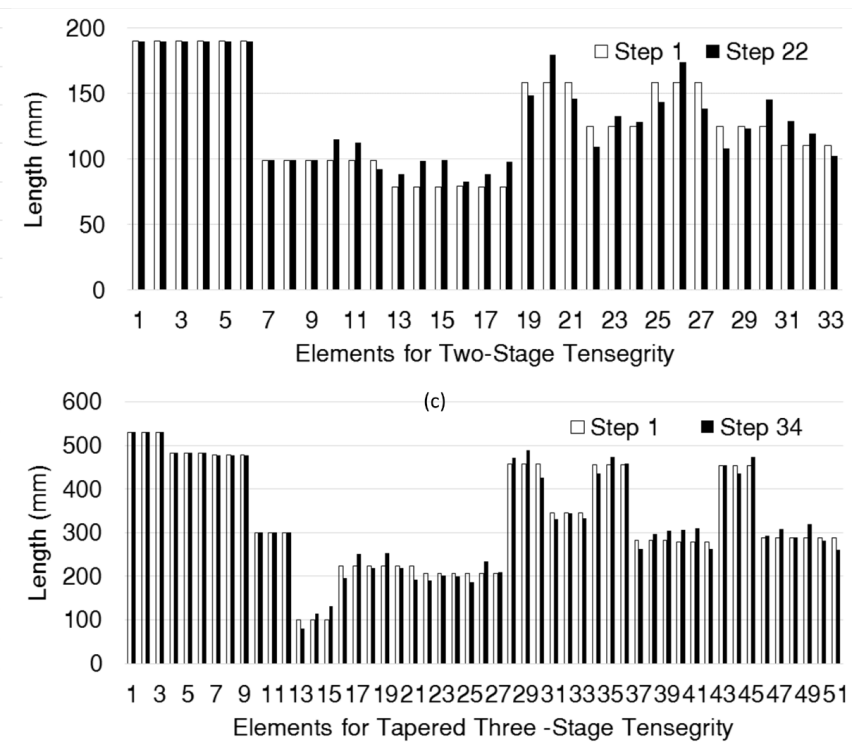

(d)

Figure 12: Length of elements for case Yp (a) simplex, (b) quadruplex, (c) two-stage and (d) tapered three-stage.

\section{CONCLUDING REMARKS}

An computational approach has been proposed for the shape change analysis of tensegrity models. The proposed approach is capable of analyzing tensegrity models which involves the following four states: initial state, deformed state, shape change state and target state. Formulation of tangent stiffness matrix of tensegrity model particularly geometrical and linear stiffness matrices, as well as the incremental equilibrium equations were presented. Use of 
optimization tool in the proposed algorithm has been shown to be able to solve the nonlinear programming problem for shape change analysis of tensegrity models. The algorithm is found to be able to search for the optimized forced elongation of cables and ensure non-slackened cables during the shape change analysis under the constraints of axial forces in the optimization process. This allows the monitored nodes to approach to the prescribed target coordinates during shape change process.

Results of four numerical examples have shown excellent convergence of the proposed algorithm for shape change analysis. In addition, it is shown that significant computational efficiency could be achieved by applying appropriate limiting value of $d_{c l}$ in the solution of the optimization problem.

Capability of tensegrity models to undergo bending, axial, twisting deformation and combinations of these deformations is also proven in the study. Results of the total computational steps suggest that the tensegrity models have better flexibility to undergo bending deformation compared to axial and torsional deformation. In addition, the study confirms the contribution of far-away nodes in contributing to the shape change. The proposed algorithm could be further expanded for use in basic study in the development of tensegrity robots capable of undergoing shape change.

\section{ACKNOWLEDGMENT}

This research was supported by Basic Science Research Program through the National Research Foundation of Korea (NRF) funded by the Ministry of Education (NRF-2019R1A2C1007548).

Author's Contributions: Conceptualization, KK Choong, T Nishimura; Methodology, T Nishimura, KK Choong, CL Oh; Investigation, CL Oh; Writing-original draft, CL Oh; Writing-review \& editing, KK Choong, CL Oh, T Nishimura, O Hassanshahi; Funding acquisition, JY Kim; Supervision, KK Choong, T Nishimura.

Editor: Pablo Andrés Muñoz Rojas.

\section{References}

Ali, N. B. H., Rhode-Barbarigos, L., Smith, I. F. (2011). Analysis of clustered tensegrity structures using a modified dynamic relaxation algorithm. International Journal of Solids and Structures 48(5): 637-647.

Averseng, J., Quirant, J., Dubé, J. F. (2012). Interactive dynamic design and analysis of tensegrity systems. International Journal of Space Structures 27(2-3): 97-105.

Caluwaerts, K., Carbajal, J. P. (2015). Energy conserving constant shape optimization of tensegrity structures. International Journal of Solids and Structures 58: 117-127.

Carpentieri, G., Skelton, R. E., Fraternali, F. (2017). A minimal mass deployable structure for solar energy harvesting on water canals. Structural and Multidisciplinary Optimization 55(2): 449-458.

Cimmino, M. C., Miranda, R., Sicignano, E., Ferreira, A. J. M., Skelton, R. E., Fraternali, F. (2017). Composite solar façades and wind generators with tensegrity architecture. Composites Part B: Engineering 115: 275-281.

Dalilsafaei, S., Eriksson, A., Tibert, G. (2012). Improving bending stiffness of tensegrity booms. International Journal of Space Structures 27(2-3): 117-129.

Du, W., Ma, S., Li, B., Wang, M., Hirai, S. (2016). Force analytic method for rolling gaits of tensegrity robots. IEEE/ASME Transactions on Mechatronics 21(5): 2249-2259.

Fazli, N., Abedian, A. (2011). Design of tensegrity structures for supporting deployable mesh an-tennas. Scientia Iranica 18(5): 1078-1087.

Furuya, H. (1992). Concept of deployable tensegrity structures in space application. International Journal of Space Structures 7(2): 143-151.

Hangai, Y., Kawaguchi, K.(1991). Shape Analysis - Generalized Inverse and Its Application. Baifukan(Tokyo). (in Japanese). 
Kim, K., Agogino, A. K., Moon, D., Taneja, L., Toghyan, A., Dehghani, B., Agogino, A. M. (2014, December). Rapid prototyping design and control of tensegrity soft robot for locomotion. In Robotics and Biomimetics (ROBIO), 2014 IEEE International Conference on (pp. 7-14). IEEE.

Li, Q., Skelton, R. E., Yan, J. (2011, July). Energy optimization of deployable tensegrity structure. In Control Conference (CCC), 2011 30th Chinese (pp. 2146-2151). IEEE.

Moored, K. W., Kemp, T. H., Houle, N. E., Bart-Smith, H. (2011). Analytical predictions, optimization, and design of a tensegritybased artificial pectoral fin. International Journal of Solids and Structures 48(22-23): 3142-3159.

Oh, C.L.(2017). Form Finding and Shape Change Analysis of Spine Inspired Bio-Tensegrity Model. PhD Thesis, School of Civil Engineering, Universiti Sains Malaysia.

Oh, C. L., Choong, K. K., Low, C. Y. (2012). Biotensegrity Inspired Robot-Future Construction Alternative. Procedia Engineering 41: $1079-1084$.

Oh, C. L., Choong, K. K., Nishimura, T., Kim, J.Y. (2016). Form-finding of Human Spine Inspired Biotensegrity. Proceedings of the IASS Annual Symposium 2016 "Spatial Structures in the 21 ${ }^{\text {st }}$ Century", ed.K.Kawaguchi, M.Ohsaki and T.Takeuchi, 26-30 September, Tokyo, Japan.

Pinaud, J. P. (2005). Deployable tensegrity towers.

Rhode-Barbarigos, L., Ali, N. B. H., Motro, R., Smith, I. F. (2012). Design aspects of a deployable tensegrity-hollow-rope footbridge. International Journal of Space Structures 27(2-3): 81-95.

Skelton, R. E., Oliveira, M. C. (2010). Optimal complexity of deployable compressive structures. Journal of the Franklin Institute 347(1): 228-256

Sultan, C., Skelton, R. (2003). Deployment of tensegrity structures. International Journal of Solids and Structures 40(18): 46374657.

Sultan, C., Skelton, R. T. (1998, July). Tendon control deployment of tensegrity structures. In Smart Structures and Materials 1998: Mathematics and Control in Smart Structures (Vol. 3323, pp. 455-467). International Society for Optics and Photonics.

Tur, J. M. M., Juan, S. H. (2009). Tensegrity frameworks: Dynamic analysis review and open problems. Mechanism and Machine Theory 44(1): 1-18.

van de Wijdeven, J., de Jager, B. (2005, June). Shape change of tensegrity structures: design and control. In American Control Conference, 2005. Proceedings of the 2005 (pp. 2522-2527). IEEE.

Veuve, N., Safaei, S. D., Smith, I. F. (2016). Active control for mid-span connection of a deployable tensegrity footbridge. Engineering Structures 112: 245-255.

Zhang, P., Kawaguchi, K.I., Feng, J.(2014). Prismatic tensegrity structures with additional cables: Integral symmetric states of self-stress and cable-controlled reconfiguration procedure. International Journal of Solids and Structures 51(25-26):42944306. 